\title{
Terapia Nutricional em Unidade de Terapia Intensiva*
}

\author{
Nutritional Therapy in Intensive Care Unit
}

\author{
lára Kallyanna Cavalcante Ferreira ${ }^{1}$
}

\section{RESUMO}

JUSTIFICATIVA E OBJETIVOS: Esta revisão tem como objetivo levantar os principais aspectos necessários para a realização de terapia nutricional segura e eficaz ao paciente crítico.

CONTEÚDO: Foi feito um levantamento bibliográfico com livros didáticos e artigos científicos em Português, Inglês e Espanhol com resultados dos últimos 20 anos.A terapia nutricional é parte integrante dos cuidados do paciente em unidades de terapia intensiva. O seu sucesso envolve as etapas de avaliação nutricional, determinação das necessidades de calorias e nutrientes, decisão da via de infusão e o tipo de dieta empregada.

CONCLUSÕES: O uso de nutrientes com a finalidade de melhorar a função imunológica (imunonutrientes), é cada vez mais freqüente, porém seu uso não está bem estabelecido para pacientes críticos. Mais estudos clínicos são necessários para estabelecer a melhor forma de nutrir o paciente crítico.

Unitermos: imunonutrição, nutrição enteral, nutrição parenteral, paciente crítico, terapia nutricional.

\footnotetext{
1. Nutricionista Graduada pela Universidade Federal de Goiás (UFG), Pós-Graduada em Clínica e Terapêutica Nutricional pelo Instituto de Pesquisa, Capacitação e Especialização - IPCE, Membro da Equipe Multidisciplinar de Terapia Nutricional (EMTN) do Hospital de Urgências de Goiânia - Dr. Valdemiro Cruz (HUGO)

\begin{abstract}
*Trabalho de Conclusão do Curso de Pós-Graduação em Clínica e Terapêutica Nutricional pelo Instituto de Pesquisa, Capacitação e Especialização - IPCE
\end{abstract}

Apresentado em 16 de novembro de 2006

Aceito para publicação em 13 de fevereiro de 2007

Endereço para correspondência:

lára Kallyanna Cavalcante Ferreira

Rua 100, n 89/302 Setor Sul

74080-140 Goiânia, GO

Fone: (62) 8435-9630

E-mail: kallyanna2@yahoo.com.br

(C)Associação de Medicina Intensiva Brasileira, 2007

\section{SUMMARY}

BACKGROUND AND OBJECTIVES: The purpose of this review is to approach the main necessary aspects for the accomplishment of safety and efficient nutritional therapy to the critically ill patient.

CONTENTS: Bibliographical survey with didactic books and scientific articles was made in Portuguese, English and Spanish with results of the last 20 years. Nutritional support is an integrant part in the care of patients in intensive care units. The success of the nutritional therapy involves the stages of nutritional assessment, determines the route of diet infusion and the calories and nutrients needs.

CONCLUSIONS: The use of nutrients with immune function (immunonutrients) is each more frequents, however, its use is not well established for critical illness. More clinical studies are necessary to establish the best form to nourish the critical ill patient.

Key Words: critical ill patient, enteral nutrition, immunonutrition, nutritional therapy, parenteral nutrition

\section{INTRODUÇÃO}

A terapia nutricional é peça fundamental nos cuidados dispensados ao paciente crítico, devido às evidências científicas que comprovam que o estado nutricional interfere diretamente na sua evolução clínica'.

O paciente desnutrido cursa mais facilmente com infecção, demora mais para cicatrizar, exige maiores cuidados intensivos e permanece internado por mais tempo no hospital e unidade de terapia intensiva (UTI)².

O paciente crítico, após a agressão, sofre uma série de alterações hormonais visando manter a homeostase hemodinâmica. Estas alterações causam, dentre outros efeitos, intolerância à glicose e catabolismo protéico elevado. A oferta de nutrientes, embora não possa reverter a proteólise, a gliconeogênese e a lipólise associadas ao estresse, pode reduzir as conseqüências do catabolismo exacerbado, melhorando a evolução clínica ${ }^{3}$. 
Fatores inerentes ao tratamento, como ventilação mecânica, uso de sedativos e fármacos vasoativos, tornam o suporte nutricional um desafio aos profissionais envolvidos. A via de administração da terapia nutricional, o tipo e a quantidade de dieta ofertada devem ser criteriosamente avaliados para diminuir o aparecimento de complicações ${ }^{4}$.

A proposta deste estudo foi abordar os principais aspectos necessários para a realização de terapia nutricional segura e eficaz ao paciente em estado grave.

O levantamento bibliográfico foi feito através de livros didáticos e artigos científicos nas linguagens portuguesa, inglesa e espanhola, via internet, nas bases de dados CAPES, MedLine e PubMed, sendo considerados os resultados publicados nos últimos 20 anos.

O objetivo deste estudo foi levantar os principais aspectos necessários para a realização de terapia nutricional segura e eficaz ao paciente crítico.

\section{AVALIAÇÃO NUTRICIONAL}

Os parâmetros utilizados na avaliação nutricional incluem avaliação clínica, antropométrica, bioquímica e imunológica 5 .

A história clínica deve abordar alterações da ingestão alimentar (inapetência, disfagia, jejum para procedimentos) e perdas excessivas (vômitos, fístulas, diarréia, má absorção) ${ }^{6}$. A avaliação física inclui o aspecto geral do paciente, observando se ele apresentava edema, ascite, caquexia, obesidade, alterações cutâneas, alterações mucosas, petéquias ou equimose, glossite, estomatite ou queilose. O sistema músculo-esquelético foi inspecionado e palpado, com atenção especial aos bíceps, tríceps, quadríceps e aos músculos interósseos das mãos ${ }^{7}$.

O dados antropométricos incluem medidas de dobras cutâneas, circunferências e divisão dos compartimentos corporais. Contudo, a utilização em pacientes internados em UTI tem valor limitado, devido a possíveis alterações na interpretação dos resultados ${ }^{8}$.

O peso seco e a altura são utilizados para calcular o peso ideal, o percentual de perda de peso e o índice de massa corporal (IMC), que é o peso dividido pela altu-

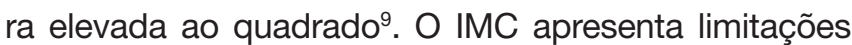
devido às alterações no peso corporal freqüentemente presentes. Valor abaixo de $18,5 \mathrm{~kg} / \mathrm{m}^{2}$ está relacionado com maior permanência em UTI, aumento de complicações pós-operatórias e dificuldade em retorno à alimentação por via oral (VO). Em geral, valores abaixo de 15 $\mathrm{kg} / \mathrm{m}^{2}$ associam-se a aumento na morbimortalidade ${ }^{8}$.
Os parâmetros de medida como dobras cutâneas, circunferência muscular do braço (CMB) e outras circunferências (braço, panturrilha) têm valor limitado na avaliação nutricional, devido à interpretação controversa dos resultados pelas alterações freqüentes no compartimento hídrico 9 .

O índice-creatinina-altura (ICA) é uma maneira laboratorial de expressar um índice antropométrico, realizado através de coleta precisa da urina de 24 horas. Devemse considerar fatores que influenciam na excreção de creatinina, como idade, dieta, imobilização prolongada, doença renal, infecção e trauma ${ }^{10}$.

A avaliação da imunidade celular e testes de sensibilidade cutânea têm boa correlação com o estado nutricional, contudo, podem ser afetados por variáveis clínicas presentes no paciente crítico, limitando o seu uso ${ }^{11}$.

Testes de função muscular são utilizados como indicadores do estado nutricional tanto de forma ativa (força muscular respiratória, capacidade de apreensão) como passiva (resposta de contração muscular à estimulação elétrica). Esses parâmetros têm os seus usos limitados por fatores como sedação e polineuropatias ${ }^{12}$.

A avaliação subjetiva global (ASG) é um método de fácil aplicação, que classifica o paciente em bem nutrido, moderadamente desnutrido ou em risco de desnutrição e desnutrido grave. Este método tem demonstrado boa sensibilidade e especificidade na identificação de desnutrição hospitalar, mas não há comprovação da sua efetividade em pacientes de UTI6.

Assim como os parâmetros antropométricos, os testes bioquímicos também sofrem alterações no paciente crítico. As proteínas hepáticas (albumina, pré-albumina, transferrina) são comumente utilizadas para avaliação do estado nutricional. Contudo, essas proteínas têm função limitada no paciente crítico, por fatores como hemodiluição e conversão da atividade hepática na síntese de proteínas de fase aguda, resultando em diminuição das proteínas viscerais. Devido a estas alterações, valores diminuídos nestes pacientes refletem mais a gravidade da doença do que o estado nutricional ${ }^{13}$.

A bioimpedância $(B \mid A)$ é um método seguro, rápido, não-invasivo e reproduzível para estimativa da massa corporal magra, e pode ser realizada na beira do leito. É uma técnica que pode ser utilizada para análise da composição corporal em indivíduos saudáveis e em muitas condições clínicas, exceto as que envolvem distúrbios na distribuição hídrica corporal. Como estes distúrbios são freqüentes em pacientes críticos, não é uma técnica recomendada ${ }^{7}$. 
É importante ressaltar que não existe um teste "padrão ouro" que seja sensível e específico para detectar desnutrição no paciente crítico, pois as alterações clínicas provocadas pelo estresse metabólico limitam a interpretação dos testes. O ideal é a combinação de diversos parâmetros na prática clínica, pois a detecção da desnutrição nas suas fases iniciais, bem como avaliações periódicas do estado nutricional são necessárias para avaliar a eficácia de qualquer intervenção nutricional ${ }^{14}$.

\section{NECESSIDADES NUTRICIONAIS}

\section{Calorias}

O fornecimento de calorias de acordo com a condição clínica é fundamental para o controle metabólico adequado do paciente crítico. O gasto energético varia de acordo com o tipo de agressão (trauma, sepse, intervenção cirúrgica), grau de atividade do paciente (ventilação espontânea ou mecânica, sedação), estágio da doença e estado nutricional prévio do paciente ${ }^{3}$.

A necessidade energética pode ser estimada ou medida diretamente. Diversos métodos de avaliação do gasto energético no paciente grave foram descritos, porém todos apresentam limitações ${ }^{15}$.

A calorimetria indireta é um método não-invasivo que mede o calor liberado durante o processo oxidativo através dos valores do consumo de oxigênio $\left(\mathrm{VO}_{2}\right)$ e produção de gás carbônico $\left(\mathrm{VCO}_{2}\right)$. Embora seja considerado atualmente o "padrão ouro" de avaliação do gasto energético, apresenta limitações técnicas de aplicação, como custo elevado do equipamento, exigência de pessoal treinado para aferição com disponibilidade de tempo e necessidade de fração de oxigênio inspirado $\left(\mathrm{FIO}_{2}\right)$ maior que 0,6 , dentre outros ${ }^{16}$.

Os métodos mais comuns para determinação das necessidades energéticas são as equações preditivas, devido à facilidade de execução e custo zero. Existem atualmente cerca de 190 fórmulas publicadas na literatura para estimativa do gasto energético, que utilizam as variáveis: peso, altura, idade, sexo e superfície corporal ${ }^{15}$.

A fórmula mais amplamente utilizada é a equação de Harris-Benedict, desenvolvida em 1919, acrescida de fatores atividade e estresse. Porém, sua acurácia é limitada, pois a fórmula é derivada de predição para indivíduos saudáveis ${ }^{17}$.

Para a estimativa por equação, é preferível o uso do peso "atual" em pacientes eutróficos e desnutridos no lugar do peso desejável, e, em obesos, deve-se utilizar o peso ajustado para obesidade ${ }^{18}$.

Em pacientes hemodinamicamente estáveis em ventilação mecânica, o uso de $120 \%$ da taxa metabólica em repouso parece suprir adequadamente as suas necessidades $^{19}$.

Outra medida para o cálculo do gasto energético é o método de Fick, que utiliza dados hemodinâmicos, como o débito cardíaco, a concentração de hemoglobina, as saturações venosa e arterial de oxigênio. Necessita ter inserido um cateter triluminal na artéria pulmonar $^{20}$.

Além das fórmulas preditivas, o cálculo direto utilizando $25 \mathrm{kcal} / \mathrm{kg} /$ dia de peso usual parece se adequar à maioria dos pacientes ${ }^{8}$.

A European Society for Parenteral and Enteral Nutrition (ESPEN) recomenda durante a fase aguda inicial, uma oferta de 20 a $25 \mathrm{kcal} / \mathrm{kg} / \mathrm{dia}$, e na fase de recuperação, 25 a $30 \mathrm{kcal} / \mathrm{kg} / \mathrm{dia}^{21}$.

Em pacientes obesos, recomenda-se ofertar de 20 a $30 \mathrm{kcal} / \mathrm{kg} / \mathrm{dia}$, com base no peso ajustado para obesidade $^{22}$.

\section{Carboidratos}

Trinta a $70 \%$ das calorias totais devem ser fornecidas na forma de carboidratos, na dose de 2 a $5 \mathrm{~g} / \mathrm{kg} / \mathrm{dia}^{8}$. A oferta de glicose deve ser ajustada para evitar níveis de glicemia $>140 \mathrm{mg} / \mathrm{dL}$, mas estudos estão sendo realizados para definir níveis ideais de glicemia para os pacientes críticos. Pode ser necessário o uso de insulina para manter os níveis de glicose próximos ao normal ${ }^{20}$.

\section{Lipídeos}

Quinze a $30 \%$ das calorias devem ser oferecidas na forma de lipídeos ${ }^{8}$. A quantidade mínima a oferecer é de $1 \mathrm{~g} / \mathrm{kg} / \mathrm{dia}$, sem exceder a $1,5 \mathrm{~g} / \mathrm{kg} / \mathrm{dia}$. Deve-se evitar o aporte excessivo de lipídeos, pois está relacionado a efeitos imunossupressivos, com aumento na incidência de infecções ${ }^{20}$.

O balanceamento dos vários tipos de ácidos graxos também é importante, pois pode influenciar vias de síntese de eicosanóides. Atualmente são utilizados no suporte nutricional, triglicerídeos de cadeia longa (TCL) pertencentes ás séries n-3, n- 6 e n-9, individualmente ou em combinação com triglicerídeos de cadeia média $(\mathrm{TCM})^{20}$.

\section{Proteínas}

Quinze a $20 \%$ do total de calorias devem ser fornecidos como proteína ou aminoácido ${ }^{8}$. Deve-se iniciar 
com aporte de 1 a $1,5 \mathrm{~g} / \mathrm{kg} / \mathrm{dia}$. Este valor pode ser aumentado em situações de maior perda protéica (queimaduras, feridas abertas, enteropatias com perda de proteínas), devendo ser ajustado de acordo com controles periódicos de balanço nitrogenado e alterações na uréia plasmática, para promover retenção nitrogenada e síntese protéica ${ }^{20}$.

A dose protéica deve ser reduzida nos casos da perda de nitrogênio urinário exceder a $100 \mathrm{mg} / \mathrm{dL}$ ou o nível de amônia sanguínea se associar à encefalopatia clínica $^{8,9}$. Em pacientes obesos, recomenda-se aporte protéico de 1,5 a 2 g/kg/dia de peso ideal ${ }^{22}$.

\section{Água, Eletrólitos e Vitaminas}

Não estão bem estabelecidas as necessidades de vitaminas, minerais e elementos-traço nos pacientes críticos. A determinação das necessidades de água e eletrólitos deve ser baseada na determinação do balanço diário destes elementos, incluindo parâmetros cardiovascular, renal, hepático e testes bioquímicos ${ }^{3}$.

Deve-se monitorar constantemente fósforo, magnésio e zinco para, se necessário, adequar a oferta a fim de manter níveis séricos normais ${ }^{8,20}$.

As calorias totais devem ser fornecidas em volume consistente com as necessidades de líquidos. Em geral, é necessário $1 \mathrm{~mL}$ de água por caloria administrada ${ }^{8}$.

\section{TERAPIA DE NUTRIÇÃO ENTERAL (TNE)}

A TNE faz parte da rotina de tratamento intensivo em pacientes impossibilitados de utilizar a via oral para alimentação que possam utilizar o trato gastrintestinal (TGI). O uso da nutrição enteral (NE) está associado a redução no número de complicações infecciosas, manutenção da integridade da barreira mucosa intestinal e redução da translocação bacteriana ${ }^{23}$.

Quanto ao posicionamento da sonda, o uso de alimentação pós-pilórica quando comparado á infusão gástrica reduz a prevalência de regurgitação, pneumonia associada à aspiração e aumento da quantidade ingerida de nutrição enteral, mas não há diferença entre os dois métodos com relação à redução da mortalidade e do tempo de internação ${ }^{24}$.

Recomenda-se sempre verificar o posicionamento da sonda no trato digestivo. Em unidades em que há dificuldade de acesso, a NE pós-pilórica deve ser considerada em pacientes com alto risco de intolerância à NE (uso de inotrópicos, sedativos, drenagem nasogástrica), ou quando há risco de regurgitação e aspiração. Em unidades em que não é possível obter o acesso intestinal, considerar a alimentação pós-pilórica estritamente para pacientes que apresentam repetidamente alto resíduo gástrico, e que não toleram quantidades adequadas de NE no estômago ${ }^{21,25}$.

Muitos pacientes críticos após lesão ou em pós-operatório desenvolvem gastroparesia, o que limita a tolerância à alimentação gástrica. Contudo, a diminuição ou ausência de ruídos intestinais são geralmente interpretados como indicativos de que o intestino delgado não está funcionando. Já é reconhecido que a função do intestino delgado e a habilidade de absorver os nutrientes permanece intacta, independente do estado crítico, presença de gastroparesia e ausência de ruídos intestinais ${ }^{8,9}$. Estas características do paciente crítico erroneamente retardam a instituição da nutrição enteral, contribuindo para balanço nitrogenado negativo e conseqüente perda de peso ${ }^{14}$.

A presença de distensão abdominal, em qualquer caso, é um sinal de alerta que, provavelmente indica incapacidade do tubo digestivo para processar os substratos, devendo-se suspender a dieta e avaliar o paciente. A dieta poderá ser reinstituída após se descartar alterações significativas?.

A aspiração pulmonar é uma complicação temida em pacientes em UTI. Muitas estratégias são utilizadas para prevenir a aspiração, como assepsia oral, posição supina, uso de sondas de fino calibre, elevação da cabeceira em $45^{\circ}$ e cuidados de enfermagem ${ }^{26}$.

Nos pacientes incapazes de tolerar a alimentação gástrica (resíduo gástrico $>150 \mathrm{~mL}$ ) o risco de aspiração é alto, devendo ser obtido acesso ao intestino delgado durante $o$ ato cirúrgico, por via endoscópica ou percutânea e descompressão gástrica simultânea, o que proporciona NE efetiva ${ }^{8,9}$.

O uso de NE precoce, isto é, com início em 24-48 horas após a admissão em UTI está relacionada a melhora do balanço nitrogenado, manutenção da função intestinal, melhora da imunidade, melhor capacidade antioxidante celular e diminuição da resposta hipermetabólica ${ }^{25}$. Embora seja recomendada para manter a perfusão e a integridade da mucosa intestinal e seja bem tolerada na maioria dos pacientes, a NE precoce pode causar isquemia e necrose, principalmente em paciente póschoque. Esta necrose isquêmica não envolve oclusão de vasos, e tem sido chamada de necrose intestinal não-oclusiva. Os mecanismos de ação propostos incluem o aumento da demanda metabólica da mucosa intestinal pela presença de nutrientes no lúmen, com diminuição da perfusão mucosa. A hipótese é que a distensão intestinal contribua para esta isquemia mu- 
cosa, e que a estase intestinal permita o acúmulo de toxinas bacterianas. Para pacientes que apresentam risco para esta complicação, a NE deve ser evitada até que o paciente esteja clinicamente estável ${ }^{27}$.

Diversos fatores podem impedir o fornecimento do total de calorias exigido para o paciente critico. A quantidade de dieta oferecida pode ser limitada por intolerância à dieta e a freqüentes procedimentos realizados na UTI (banho, fisioterapia, extubação, etc.). Estudos apontam um oferecimento energético abaixo do necessário em muitos pacientes, podendo piorar a desnutrição e agravar o quadro clinico ${ }^{28}$. Protocolos de NE parecem ajudar a efetivar as praticas de alimentação e limitar interrupções desnecessárias ${ }^{25}$.

Recomenda-se iniciar a terapia nutricional com fórmulas com proteína intacta (polimérica). Fórmulas à base de peptídeos podem beneficiar pacientes com complicações gastrintestinais (síndrome do intestino curto, pancreatite, etc.), sendo necessários mais estudos para definir a sua recomendação ${ }^{21,25}$.

A instalação de traqueostomia é uma oportunidade para iniciar a dieta via oral em pacientes dependentes de ventilação mecânica (VM). Porém, muitos pacientes que permanecem longos períodos ventilados por traqueostomia apresentam disfagia, pois os tubos fixados na laringe impedem a coordenação do movimento de deglutição, alterando o fechamento da glote. O ideal é avaliar e tratar a disfagia com um protocolo e um fonoaudiólogo, durante a reintrodução da dieta por via oral $^{29}$.

\section{NUTRIÇÃO PARENTERAL (NP)}

Em geral, a NP é indicada a pacientes impossibilitados de utilizar o trato gastrintestinal durante sete a 10 dias, que apresentem perda de peso superior a $10 \%$ do usual, incapazes de tolerar a NE ou quando contra-indicado o seu uso e que não apresentem doença terminal ${ }^{7}$.

O uso de NP está relacionado com maior número de complicações, inclusive na via de acesso e ao custo elevado. As formulações parenterais não são tão completas quanto as enterais, porém a meta nutricional é atingida com maior facilidade por via parenteral ${ }^{\text {. }}$.

A administração de NP é contra-indicada em pacientes hemodinamicamente instáveis (choque séptico, cardiogênico, hipovolemia), edema agudo de pulmão, anúricos sem diálise e na presença de distúrbios eletrolíticos e metabólicos graves ${ }^{30}$.

Infecção no local do cateter é uma complicação co- mum que pode levar a sepse, associada a aumento da morbidade, mortalidade e maiores custos. O tratamento da sepse envolve remoção do cateter e antibioticoterapia apropriada. Protocolos de inserção e cuidados com cateteres devem ser implantados para prevenir complicações ${ }^{7}$.

A NP deve iniciar com 100 a $150 \mathrm{~g}$ de glicose, e baixas concentrações de cloreto de sódio $(\mathrm{NaCl})$, sendo realizada a monitorização estrita de eletrólitos (diariamente nos primeiros 2 a 3 dias) e controle glicêmico (a cada 6 horas até normalizar valores de glicose) ${ }^{8}$.

A infusão de glicose estimula a secreção de insulina adicional e tem um efeito antilipolítico. A hiperglicemia limita a quantidade ofertada de glicose e o grau da hiperglicemia induzida pela NPT é diretamente proporcional à dose de glicose infundida e ao grau de lesão ${ }^{23}$. Muitos pacientes necessitam utilizar insulina regular junto à administração de NPT, como componente da fórmula ou com administração subcutânea suplementar.

Uma maneira de prevenir a hiperalimentação é pela análise do quociente respiratório (QR). Valores maiores que 1 geralmente indicam hiperalimentação. Valores entre 0,8 e 1 indicam produção elevada de gás carbônico 8 .

Os lipídios são administrados na forma de emulsão. Inicialmente as emulsões lipídicas eram unicamente à base de soja, contendo somente ácidos graxos de cadeia longa (AGCL). Estudos demonstraram que as emulsões lipídicas à base de soja, ricas em ácidos graxos poliinsaturados (PUFA) $n-6$ afetavam negativamente os sistemas imunes, relacionando o excesso de $n-6$ e a baixa quantidade de n-3 em maior risco de peroxidação, com alteração na função de neutrófilos, linfócitos, monócitos e macrófagos. A descoberta destas alterações estimulou o desenvolvimento de novas composições comerciais, com diferentes tipos de ácidos graxos ${ }^{31}$.

Os triglicerídeos de cadeia média (TCM) apresentam hidrólise mais fácil e rápida que os triglicerídeos de cadeia longa (TCL), sendo produzidas emulsões comerciais com mistura TCL/TCM (soja e coco, respectivamente) na relação 50:5032. Contudo, esta mistura também afeta a função dos neutrófilos. Recentemente foram desenvolvidas emulsões à base de óleo de oliva e soja, com baixa concentração de poliinsaturados (PUFA) e ricos em ácidos graxos monoinsaturados (MUFA), que apresentam menos efeitos inibitórios do sistema imune, com boa tolerância e preservação da função hepática ${ }^{33}$.

Emulsões contendo óleo de peixe aumentam a incorporação de n-3 nas membranas celulares, e não pre- 
judicam a coagulação e a função plaquetária. Estas emulsões parecem preservar a função imune e prevenir alguns aspectos da resposta inflamatória, com redução do tempo de internação no hospital e na UTI ${ }^{31}$. Os aminoácidos são incluídos na NPT como fonte de nitrogênio para a síntese protéica. A dose de proteína deve ser ajustada com monitorização periódica, mas não deve ser excessiva pelo risco de azotemia ${ }^{8}$.

A administração de aminoácidos essenciais (AAE) para pacientes com insuficiência renal ${ }^{34}$ e aminoácidos de cadeia ramificada (AACR) em pacientes com insuficiência hepática ${ }^{35}$ não tem demonstrado efeito positivo quando comparados ao uso de solução-padrão.

$A$ vitamina $K$ não faz parte das multivitaminas presentes na solução de NP, devido a alterações que podem causar em pacientes recebendo anticoagulantes. Portanto, pacientes em uso de NPT que não fazem terapia anticoagulante devem receber vitamina $\mathrm{K}$ suplementar ${ }^{36}$.

As necessidades de sódio e potássio são muito variáveis, sendo adicionados à NP de acordo com as necessidades individuais. O conteúdo de cloreto e acetato deve ser ajustado para manter o balanço ácido-base, geralmente com quantidades iguais, mas podem exigir ajustes individuais. As quantidades de magnésio, cálcio e fósforo devem ser baseados nas necessidades, sendo suplementados quando necessário. Ferro não é adicionado à solução de NPT, devendo ser administrado por via venosa ${ }^{36}$.

\section{NUTRICÃO ENTERAL ASSOCIADA À NUTRICÃO PARENTERAL}

Quando a necessidade nutricional não é atingida com o uso de nutrição enteral (NE), a suplementação com nutrição parenteral (NP) é uma possibilidade. A associação da NE e NP têm sido analisadas em duas vertentes, quando a NE e NP são iniciadas simultaneamente e a NP é interrompida quando o paciente tolera totalmente a NE ou quando a NP é introduzida apenas após alguns dias de NE, quando confirmada a intolerância à NE.

A análise de estudos comparando a introdução simultânea de NE e NP demonstrou aumento na mortalidade quando comparado ao uso de NE isolada. A NP suplementar não foi associada a aumento na incidência de infecções e tempo de internação e ventilação mecânica. Mesmo quando excluídos da análise os pacientes hiperalimentados, os resultados permaneceram, demonstrando que a alta mortalidade da associação não está relacionada à hiperalimentação ${ }^{25}$.
Em pacientes que não conseguem atingir suas necessidades somente com a NE, os benefícios da associação parecem sobrepor os riscos potenciais, sendo recomendadas as suplementações com NP. Deve-se ter cuidado, contudo, para que a NP não exceda à necessidade energética do paciente, causando hiperalimentação, devendo haver controle metabólico rigoroso ${ }^{21}$.

\section{CONTROLE GLICÊMICO}

A hiperglicemia é uma reação natural do organismo ao estresse metabólico, devido às alterações hormonais. Além disso, os cuidados ao paciente crítico aumentam a resposta hiperglicêmica, com o uso de corticosteróides, agentes adrenérgicos e suporte nutricional rico em glicose. Apesar de ser uma resposta normal do organismo, a redução dos níveis de glicemia melhora a evolução e diminuem o risco de complicações, especialmente infecciosas ${ }^{37}$.

Van den Bergue e col. ${ }^{38}$ em estudo prospectivo aleatório em UTI cirúrgica avaliou o controle glicêmico estrito através de protocolo de infusão contínua de insulina para manter níveis de glicose abaixo de 110 $\mathrm{mg} / \mathrm{dL}$, e observaram redução da morbidade e mortalidade, associados a redução da bacteremia, necessidade de diálise, transfusão, ventilação mecânica prolongada e polineuropatia.

Em recente estudo prospectivo aleatório, Van den Berg e col. ${ }^{39}$ analisaram o uso de protocolo de terapia insulínica intensiva em UTI médica. O resultado demonstrou redução da morbidade, mas não da mortalidade. O risco de morte e doença foi reduzido em pacientes tratados por mais de três dias, contudo, estes pacientes não podem ser identificados no momento da admissão. A redução da morbidade resultou de prevenção de doença renal, desmame mais rápido da VM e menor tempo de UTI e internação.

A terapia insulínica tem demonstrado ser promissora para uso de rotina em UTI. Mais estudos são necessários para definir faixas ideais de glicemia em diferentes situações clínicas.

\section{IMUNONUTRIÇÃO}

Apesar dos avanços terapêuticos para tratamento do paciente crítico, infecção, sepse e falência de múltiplos órgãos ainda são a maior causa de mortalidade, eventualmente associado à imunossupressão. Pela forte relação entre nutrição e imunidade publicados na literatura clínica, o uso de nutrientes específicos visan- 
do a restauração e manutenção da resposta imune é cada vez mais freqüente, tanto com nutrientes isolados quanto em formulações. As dietas imunomoduladoras têm como principais componentes adicionados: arginina, ácidos graxos n-3, glutamina, nucleotídeos, micronutrientes e vitaminas antioxidantes ${ }^{40}$.

O aminoácido arginina, classificado como condicionalmente essencial em estados de estresse, exerce papel na síntese protéica, como substrato para o ciclo da uréia e produção de óxido nítrico. É também um secretagogo para hormônio de crescimento, prolactina e insulina ${ }^{41}$.

$\mathrm{O}$ uso de fórmula suplementada com arginina tem apresentado benefícios a pacientes cirúrgicos, com redução da taxa de infecção. Porém, em pacientes críticos com sepse e infecção grave, este efeito não acontece ${ }^{42}$. A análise de ensaios clínicos bem conduzidos com pacientes críticos em geral não apontou diferenças nas taxas de mortalidade e infecção com o uso de fórmulas enriquecidas com arginina. Porém, quando analisada em população específica de pacientes com sepse, os grupos que receberam fórmula com arginina apresentaram risco potencial, com aumento na taxa de mortalidade quando comparado ao uso de fórmula padrão ${ }^{21,25}$. Uma possível explicação é que o uso da arginina pode aumentar a liberação de citocinas pró-inflamatórias e óxido nítrico, com aumento da resposta inflamatória. Nestes pacientes a administração de fórmulas ricas em arginina pode causar hipotensão temporária, aumento no débito cardíaco e diminuição na resistência vascular e pulmonar sistêmica. Devido a arginina intensificar a resposta inflamatória, os efeitos tóxicos são maiores em pacientes com sepse, síndrome da resposta inflamatória sistêmica (SRIS) ou infecção grave ${ }^{42}$. Devido ao risco potencial, associado ao uso de dietas suplementadas com arginina em pacientes com choque e sepse, o seu uso não é recomendado nesses pacientes ${ }^{21,25}$.

A glutamina é o aminoácido mais abundante no plasma. Está envolvido em diversos processos bioquímicos e metabólicos, o que o torna condicionalmente essencial em estados catabólicos. É o substrato principal de células de proliferação rápida, como enterócitos e células imunes. Estudos clínicos em pacientes críticos e cirúrgicos têm indicado que a suplementação de glutamina diminuiu a taxa de complicações infecciosas ${ }^{43}$. O uso de glutamina apresenta benefícios a pacientes com trauma e queimaduras, está relacionado a menor número de complicações infecciosas e redução do tempo de internação ${ }^{44}$. Não há evidência científica suficiente para recomendar o uso rotineiro de gluta- mina em pacientes cirúrgicos ou pacientes críticos heterogêneos ${ }^{21,25}$.

O uso da glutamina tem demonstrado efeitos benéficos em pacientes críticos quando suplementado em fórmulas de NP, com diminuição da mortalidade, sendo recomendada a sua suplementação. Não há dados que demonstrem melhora na taxa de infecção e tempo de internação ${ }^{21,25}$.

Os ácidos graxos n-3 diminuem a produção de citocinas inflamatórias e eicosanóides, com diminuição da resposta inflamatória e da imunossupressão ${ }^{45}$. Sua degradação, diferente dos ácidos graxos n-6, leva à formação de moléculas vasodilatadoras, reduz a produção de ubiquitina (principal proteína indutora de proteólise), podendo ter um efeito inibidor do catabolismo protéico no paciente crítico. Por estas propriedades, pode ser utilizado com fins terapêuticos em vários estados inflamatórios crônicos e agudos ${ }^{46}$.

Os nucleotídeos da dieta são essenciais na imunidade célula-mediada. Sua presença é especialmente importante durante o desenvolvimento, maturação e reparo intestinal. Suplementos de nucleotídeos podem ajudar a preservar a estrutura e função intestinal durante o uso de NP. Sua carência pode ter efeitos similares aos da carência de glutamina na barreira mucosa intestinal e na função de absorção. Contudo, as implicações quanto às vias de administração, a composição e a dose permanecem indefinidas, bem como o benefício clínico na alimentação do paciente crítico não está comprovada ${ }^{47}$.

O estado crítico é associado à formação de radicais livres de oxigênio e diminuição da capacidade antioxidante, levando ao estresse oxidativo. Acredita-se que o suprimento exógeno de determinadas vitaminas e elementos-traço podem ajudar a balancear os níveis de oxidantes e antioxidantes no paciente crítico ${ }^{48}$. Doses de selênio, zinco, vitaminas, C, E e beta-caroteno para pacientes críticos ainda não estão estabelecidas, necessitando de maior investigação clínica ${ }^{9}$.

O efeito dos vários nutrientes varia dependendo do fundamento fisiopatológico do quanto e como os substratos influem na função imune celular e/ou na síntese de mediadores inflamatórios e/ou geração de radicais livres ${ }^{49}$. O maior estudo sobre dieta com nutrientes imunomoduladores em UTI geral, realizado por Kieft e col. ${ }^{50}$, analisou o efeito de fórmula imunonoduladora comparando ao uso de fórmula padrão em 597 pacientes, e não apresentou benefícios ao grupo de pacientes que receberam a fórmula enriquecida.

É plausível que muitos destes nutrientes testados in- 
dividualmente tenham efeitos terapêuticos (positivos ou negativos) em grupos de pacientes, mas quando combinados, seu uso deve ser cauteloso, devido à heterogeneidade de estados clínicos e variações na gravidade da doença ${ }^{49}$.

Há necessidade de se estabelecer novos paradigmas com o foco em nutrientes isolados, administrados em populações homogêneas de pacientes em estudos clínicos rigorosos.

\section{REFERÊNCIAS}

01. Klein S, Kinney J, Jeejeebhoy K et al - Nutrition support in clinical practice: review of published data and recommendations for future research directions. J Parenter Enteral Nutr. 1997;21:133-156.

02. Dempsey DT, Mullen JL, Buzby GP - The link between nutritional status and clinical outcome: can nutritional intervention modify it? Am j Clin Nutr. 1988;47:(Suppl2):352-356.

03. Atkinson M, Worthley LI - Nutrition in the critically ill patient: part I. Essential physiology and patophysiology. Crit Care Resusc, 2003;5:109-120.

04. Spain DA - When is the seriously ill patient ready to be fed? J Parenter Enteral Nutr, 2002;26:(Suppl6):S62-S68.

05. Sabol VK - Nutrition assessment of the critically ill adult. AACN Clin Issues, 2004;15:595-606.

06. BolesJ-M - Assessing Nutritional Status, em: Webb AR, Shapiro M, Singer $\mathrm{M}$ et al - Oxford Textbook of Critical Care. $1^{\text {st }}$ Ed, New York: Oxford University Press, 1999;380-382.

07. Guidelines for use of parenteral and enteral nutrition in adult and pediatric patients. ASPEN Board of Directors $\mathrm{J}$ Parenter Enteral Nutr, 2002;26:(Suppl1):1SA-138SA.

08. Cerra FB, Benitez MR, Blackburn GL et al - Applied nutrition in ICU patients. A consensus statement of the American College of Chest Physicians. Chest. 1997;111:769-778.

09. Chan S, McCowen KC, Blackburn GL - Nutrition management in the ICU. Chest 1999;115:(Suppl5):145S-148S

10. Bottoni A, Oliveira GPC, Ferrini MT et al - Avaliação Nutricional: Exames Laboratoriais, em: Waitzberg DL - Nutrição Oral, Enteral e Parenteral na

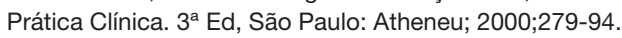

11. Dominioni L, Dionigi R - Immunological function and nutritional assessment. J Parenter Enteral Nutr, 1987;11:(Suppl5):70S-72S.

12. Escribano JA, Gomez-Tello V, Santana SR - Valoración Del estado nutricional em el paciente grave. Nutr Hosp. 2005;20:(Suppl2):5-8.

13. Escribano JA, Gomez-Tello V, Santana SR - The role of visceral proteins in the nutritional assessment of intensive care unit patients. Curr Opin Clin Nutr Metabol Care, 2003;6:211-216.

14. Jolliet $\mathrm{P}, \mathrm{Pichard} \mathrm{C}$, Biolo $\mathrm{G}$ et.al - Enteral nutrition in intensive care patients: a practical approach. Working Group on Nutrition and Metabolism, ESICM. European Society of Intensive Care Medicine. Intensive Care Med, 1998;24:848-859.

15. Silva SRJ, Waitzberg DL - Gasto Energético, em: Waitzberg DL - Nutrição Oral, Enteral e Parenteral na Prática Clínica. $3^{\mathrm{a}}$ Ed, São Paulo: Atheneu, 2000;327-342.

16. Basile-Filho A, Martins MA, Batiston MT et al - Gasto energético em pacientes sépticos: correlação entre a calorimetria indireta e as equações preditivas derivadas a partir de dados hemodinâmicos. RBTI, 2003;15:101-107.

17. O'Leary-Kelley CM,Puntillo KA, Barr $\mathrm{J}$ et al - Nutritional adequacy in patients receiving mechanical ventilation who are fed enterally. Am J Crit Care, 2005;14:222-231.

18. Oliveira RMC, Amaral ACK-B, Batista MC - Terapia Nutricional: Avaliação do Gasto Energético, em: Oliveira RMC, Cal, RGR - Terapia Intensiva. Nutrição. São Paulo: Atheneu, 2005;19-26.

19. Kan MN, Chang HH, Sheu WF et al - Estimation of energy requirements for mechanically ventilated, critical ill patients using nutritional status. Crit Care, 2003;7:R108-R115.

20. Leyba CO, Gomez-Tello V, Arbeloa CS - Requerimientos de macronutrientes y micronutrientes. Nutr Hosp, 2005;20:(Suppl2):13-17.
21. Kreymann KG, Berger MM, Deutz NE et al - ESPEN Guidelines on Enteral Nutrition: Intensive care. Clin Nutr, 2006;25:210-223.

22. El-Solh AA - Clinical approach to the critically ill, morbidly obese patient. Am J Respir Crit Care Med, 2004;169:557-561.

23. Weissman C - Nutrition in the intensive care unit. Crit Care, 1999;3:R67 R75.

24. Heyland DK, Drover JW, Dhaliwal R et al - Optimizing the benefits and minimizing the risks of enteral nutrition in the critically ill: role of small bowel feeding. J Parenter Enteral Nutr, 2002;26:(Suppl6):S51-S57.

25. Heyland DK, Dhaliwal R, Drover JW et al - Canadian clinical practice guidelines for nutrition support in mechanically ventilated, critically ill adult patients. J Parenter Enteral Nutr, 2003;27:355-373.

26. DiSario JA - Future considerations in aspiration pneumonia in the critically ill patient: what is not know, areas for future research, and experimental methods. J Parenter Enteral Nutr, 2002;26:(Suppl6):S75-S79.

27. Bernard AC, Magnuson B, Tsuei BJ et al - Defining and assessing tolerance in enteral nutrition. Nutr Clin Pract, 2004;19(5):481-486.

28. Binnekade JM, Tepaske R, Bruynzeel $P$ et al - Daily enteral feeding practice on the ICU: attainment of goals and interfering factors. Crit Care, 2005;9:R218-R225.

29. Heffner JE - Management of the chronically ventilated patient with tracheostomy. Chron Respir Dis, 2005;2:151-161.

30. Cal RGR, Monte JCM, Oliveira RMC et al - Terapia Nutricional Parenteral: Princípios, Formulação e Monitoramento, em: Oliveira RMC, Cal, RGR - Terapia intensiva. Nutrição. São Paulo: Atheneu; 2005;113-123.

31. Waitzberg DL, Torrinhas RS, Jacintho TM - New parenteral lipid emulsions for clinical use. J Parenter Enteral Nutr, 2006;30:351-367.

32. Llop J, Vuelta M, Sabin $P$ et al - Triglicérideos y nutrición parenteral. Endocrinol Nutr, 2005;52:290-296.

33. Buenestrado A et al - Olive oil-based lipid emulsion's neutral effects on neutrophil functions and leukocyte endothelial cell interactions. J Parenter Enteral Nutr, 2006;30:286-296.

34. Jimenez Jimenez FJ, Martinez JL et al - Nutrición artificial en la insuficiencia renal aguda. Nutr Hosp., 2005;20:18-21.

35. Jimenez Jimenez FJ, Gonzalez JCM et al - Nutrición artificial en la insuficiencia hepática. Nutr Hosp, 2005;20:22-24

36. Safe practices for parenteral nutrition formulation. National advisory group on standards and practice guidelines for parenteral nutrition. J Parenter Enteral Nutr, 1998;22:49-66.

37. Nasraway SAJ - Hyperglycemia during critical ilness. J Parenter Entera Nutr, 2006;30:254-258.

38. Van den Berghe $\mathrm{G}$ et al - Intensive insulin therapy in critically ill patients. N Engl J Med, 2001; 345:1359-1367.

39. Van den Bergue $\mathrm{G}$ et al - Insulin therapy in the medical ICU. N Engl J Med, 2006;354:449-461.

40. Waitzberg DL, Lotierzo PHP, Duarte AJS et al - Imunonutrição, em: Waitzberg DL - Nutrição Oral, Enteral e Parenteral na Prática Clínica. $3^{a}$ Ed, São Paulo: Atheneu; 2000;1511-1538.

41. Grant JP - Nutrition support in critically ill patients. Ann Surg, 1994;220:610-616.

42. Stechmiller JK, Childress B, Porter T - Arginine immunonutrition in critically ill patients: a clinical dillemma. Am J Crit Care, 2004;13:17-23.

43. Coaffier $M$, Dechelotte $P$ - The role of glutamine in intensive care unit patients: mechanisms of action and critical outcome. Nutr Rev, 2005;63:65-69.

44. García-de-Lorenzo A et al - Clinical evidence for enteral nutritional support with glutamine: a systematic review. Nutrition, 2003;19:805-811.

45. Alvarez W, Mobarhan S - Finding a place for immunonutrition. Nutr Ver, 2003;61:214-218.

46. Amaral ACK-B, Oliveira RMC, Cal RGR et al - Imunonutrição em Terapia Intensiva, em: Oliveira RMC, Cal, RGR - Terapia intensiva. Nutrição. São Paulo: Atheneu; 2005;239-245.

47. Mc Cauley R, Kong SF, Hall J - Glutamine and nucleotide metabolism within enterocytes. J Parenter Enteral Nutr, 1998;22:105-111.

48. Heyland DK, Dhaliwal R, Suchner $U$ et al - Antioxidant nutrients: a systematic review of trace elements and vitamins in the critically ill patients. Intensive Care Med, 2005;31:327-337.

49. Heyland D, Dhaliwal R - Immunonutrition in the critically ill: from old approaches to new paradigms. Intensive Care Med, 2005;31:501-503.

50. Kieft H, Roos AN, van Drunen JD et al - Clinical outcome of immunonutrition in a heterogenous intensive care population. Intensive Care Med, 2005;31:524-532. 